\title{
An Efficient Rigorous Approach for Identifying Statistically Significant Frequent Itemsets
}

\author{
Adam Kirsch* \\ Harvard University \\ Geppino Puccis \\ University of Padova
}

\author{
Michael Mitzenmacher ${ }^{\dagger}$ \\ Harvard University
}

\author{
Eli Upfal
Brown University
}

\author{
Andrea Pietracaprina $a^{\ddagger}$ \\ University of Padova \\ Fabio Vandin" \\ University of Padova
}

\begin{abstract}
As advances in technology allow for the collection, storage, and analysis of vast amounts of data, the task of screening and assessing the significance of discovered patterns is becoming a major challenge in data mining applications. In this work, we address significance in the context of frequent itemset mining. Specifically, we develop a novel methodology to identify a meaningful support threshold $s^{*}$ for a dataset, such that the number of itemsets with support at least $s^{*}$ represents a substantial deviation from what would be expected in a random dataset with the same number of transactions and the same individual item frequencies. These itemsets can then be flagged as statistically significant with a small false discovery rate.
\end{abstract}

Our methodology hinges on a Poisson approximation to the distribution of the number of itemsets in a random dataset with support at least $s$, for any $s$ greater than or equal to a minimum threshold $s_{\min }$. We obtain this result through a novel application of the Chen-Stein approximation method, which is of independent interest. Based on this approximation, we develop an efficient parametric multi-hypothesis test for identifying the desired threshold $s^{*}$. A crucial feature of our approach is that, unlike most previous work, it takes into account the entire dataset rather than individual discoveries. It is therefore better able to distinguish between significant observations and random fluctuations. We present extensive experimental results to substantiate the effectiveness of our methodology.

Categories and Subject Descriptors. H2.8 [Database Applications]: Data Mining

General Terms. Algorithms, Measurement

Keywords. frequent itemset mining; statistical significance; multi-hypothesis test; Poisson approximation; False Discovery Rate

\section{Introduction}

The discovery of frequent itemsets in transactional datasets is a fundamental primitive that arises in the mining of association rules and in many other scenarios [16,27]. In its original formulation, the problem requires that given a dataset $\mathcal{D}$ of transactions over a set of items $\mathcal{I}$, and a support threshold $s$, all itemsets $X \subseteq \mathcal{I}$ with support at least $s$ in $\mathcal{D}$ (i.e., contained in at least $s$ transactions) be returned. These high-support itemsets are referred to as frequent itemsets.

Since the pioneering paper by Agrawal et al. [2], a vast literature has flourished proposing variants of the problem, studying foundational issues, and presenting novel algorithmic strategies or clever implementations of known strategies (see, e.g., [12,13]), but many problems remain open [15]. In particular, assessing the significance of the discovered item- 
sets, or equivalently, flagging statistically significant discoveries with a limited number of false positive outcomes, is still poorly understood and remains one of the most challenging problems in this area.

The classical framework requires that the user decide what is significant by specifying the support threshold $s$. Unless specific domain knowledge is available, the choice of such a threshold is often arbitrary [16,27], and may lead to a large number of spurious discoveries (false positives) that would undermine the success of subsequent analysis.

In this paper, we develop a rigorous and efficient novel approach for identifying statistically significant frequent itemsets. Specifically, we flag as significant a population of itemsets extracted with respect to a certain threshold, if some global characteristics of the population deviate considerably from what would be expected if the dataset were generated with no correlations between individual items. We also enforce that the returned family of significant itemsets feature a small False Discovery Rate (FDR) [4].

\subsection{The model}

The significance of a discovery in our framework is assessed based on its deviation from what would be expected in a random dataset in which individual items are placed independently in transactions. Formally, let $\mathcal{D}$ be a dataset of $t$ transactions on a set $\mathcal{I}$ of $n$ items, where each transaction is a subset of $\mathcal{I}$. Let $n(i)$ be the number of transactions that contain item $i$ and let $f_{i}=n(i) / t$ be the frequency of item $i$ in the dataset. The support of an itemset $X \subseteq \mathcal{I}$ is defined as the number of transactions that contain $\bar{X}$. Among all possible $\left(\begin{array}{l}n \\ k\end{array}\right)$ itemsets of size $k$ ( $k$-itemsets) we are interested in statistically significant ones, that is, itemsets whose supports are significantly higher, in a statistical sense, than their expected supports in a dataset where individual items are placed independently in transactions.

Following [25], we consider a probability space of datasets with the same number of transactions $t$, on the same set of items $\mathcal{I}$ as $\mathcal{D}$, and in which item $i$ is included in any given transaction with probability $f_{i}$, independent of all other items and all other transactions. A similar model is used in [22] and [23] to evaluate the running time of algorithms for frequent itemsets mining. Let $\hat{\mathcal{D}}$ denote a random dataset from this probability space. For a given itemset $X$, the null hypothesis $H_{0}$ is that its support $s(X, \mathcal{D})$ in $\mathcal{D}$ is drawn from the same distribution as its support $s(X, \hat{\mathcal{D}})$ in $\hat{\mathcal{D}}$. The alternative hypothesis $H_{1}$ is that $s(X, \mathcal{D})$ is not drawn from that distribution, and in particular that there is a positive correlation between the occurrences of the individual items in $X$.

An alternative probability space of datasets, proposed in [11], considers all arrangements of $n$ items to $m$ transactions which match the exact item frequencies and transaction lengths as $\mathcal{D}$. Conceivably, the technique of this paper could be adapted to this latter model as well.

\subsection{Multi-hypothesis testing}

To demonstrate the importance of correcting for multiplicity of hypotheses, consider a simple real dataset of 1,000,000 transactions over 1,000 items, each with frequency 1/1000. Assume that we observed that a pair of items $(i, j)$ appears in at least 7 transactions. Is the support of this pair statistically significant? To evaluate the significance of this discovery we consider a random dataset where each item is included in each transaction with probability $1 / 1000$, independent of all items. The probability that the pair $(i, j)$ is included in a given transaction is $1 / 1,000,000$, thus the expected number of transactions that include this pair is 1. A simple calculation shows that the probability that $(i, j)$ appears in at least 7 transactions is about 0.0001 . Thus, it seems that the support of $(i, j)$ in the real dataset is statistically significant. However, each of the 499,500 pairs of items has probability 0.0001 to appear in at least 7 transactions in the random dataset. Thus, even under the assumption that items are placed independently in transactions, the expected number of pairs with support at least 7 is about 50. If there were only about 50 pairs with support at least 7 , returning the pair $(i, j)$ as a statistically significant itemset would likely be a false discovery since its frequency would be better explained by random fluctuations in observed data. On the other hand, assume that the real dataset contains 300 disjoint pairs each with support at least 7 . The probability of that event in the random dataset is less than $2^{-300}$. Thus, it is very likely that the support of most of these pairs would be statistically significant. A discovery process that does not return these pairs will result in a large number of false negative errors. Our goal is to design a rigorous methodology which is able to distinguish between these two scenarios.

In a simple statistical test a null hypothesis $H_{0}$ is tested against an alternative hypothesis $H_{1}$. A test consists of a rejection (critical) region $C$ such that if the statistic (outcome) of the experiment is in $C$ the null hypothesis is rejected, and otherwise the null hypothesis is not rejected. The significance level of a test, $\alpha=\operatorname{Pr}$ (Type I error), is the probability of rejecting $H_{0}$ when it is true (false positive). The power of the test, $1-\operatorname{Pr}$ (Type II error), is the probability of correctly rejecting the null hypothesis. The $p$-value of a test is the probability of obtaining an outcome at least as extreme as the one that was actually observed, under the assumption that $H_{0}$ is true.

In a multi-hypothesis statistical test, the outcome of an experiment is used to test simultaneously a number of hypotheses. In the context of frequent itemsets, if we test for significant itemsets of size $k$, then we are testing simultaneously $\left(\begin{array}{l}n \\ k\end{array}\right)$ null hypotheses. Each null hypothesis corresponds to the support of a given itemset not being statistically significant. A natural generalization of the significance level to multi-hypothesis testing is the Family Wise Error Rate (FWER), which is the probability of incurring at least one Type I error in any of the individual tests. If we have $m$ simultaneous tests and we want to bound the FWER by $\alpha$, then the Bonferroni method tests each null hypothesis with significance level $\alpha / m$. While controlling the FWER, this method is too conservative in that the power of the test is 
too low, giving many false negatives. There are a number of techniques that improve on the Bonferroni method, but for large numbers of hypotheses all of these techniques lead to tests with low power (see [8] for a good review).

The False Discovery Rate (FDR) was suggested by Benjamini and Hochberg [4] as an alternative, less conservative approach to control errors in multiple tests. Let $V$ be the number of Type I errors in the individual tests, and let $R$ be the total number of null hypotheses rejected by the multiple test. Then we define FDR to be the expected ratio of erroneous rejections among all rejections, namely FDR $=E[V / R]$, with $V / R=0$ when $R=0$. Designing a statistical test that controls for FDR is not simple, since the FDR is a function of two random variables that depend both on the set of null hypotheses and the set of alternative hypotheses. Building on the work of [4], Benjamini and Yekutieli [5] developed a general technique for controlling the FDR in any multi-hypothesis test (see Theorem 3).

\subsection{Our Results}

In this paper we address the classical problem of mining frequent itemsets with respect to a certain minimum support threshold, and provide a rigorous methodology to establish a threshold that can guarantee, in a statistical sense, that the returned family of frequent itemsets contains significant ones with a limited false discovery rate. Our methodology crucially relies on the following Poisson approximation result, which is the main theoretical contribution of the paper.

Consider a dataset $\mathcal{D}$ of $t$ transactions on a set $\mathcal{I}$ of $n$ items and let $\hat{\mathcal{D}}$ be a corresponding random dataset according to the our random model described in Section 1.1. Let $Q_{k, s}$ be the number of itemsets of size $k$ with support at least $s$ with respect to $\mathcal{D}$, and let $\hat{Q}_{k, s}$ be the corresponding random variable for $\hat{\mathcal{D}}$. We show that there exists a minimum support value $s_{\min }$ (which depends on the parameters of $\mathcal{D}$ and on $k$ ), such that for all $s \geq s_{\min }$ the distribution of $\hat{Q}_{k, s}$ is well approximated by a Poisson distribution. Our result is based on a novel application of the Chen-Stein Poisson approximation method [3].

The minimum support $s_{\min }$ provides the grounds to devise a rigorous method for establishing a support threshold for mining significant itemsets, both reducing the overall complexity and improving the accuracy of the discovery process. Specifically, for a fixed itemset size $k$, we test a small number of support thresholds $s \geq s_{\min }$, and, for each such threshold, we measure the $p$-value corresponding to the null hypothesis $H_{0}$ that the observed value $Q_{k, s}$ comes from a Poisson distribution of suitable expectation. From the tests we can determine a threshold $s^{*}$ such that, with user-defined confidence level $\alpha$, the number of itemsets with support at least $s^{*}$ is not sampled from a Poisson distribution and is therefore statistically significant. The fact that the number of itemsets with support at least $s^{*}$ is statistically significant does not imply necessarily that each of the itemsets is significant. However, our test is also able to guarantee a user-defined upper bound $\beta$ on the expected ratio of false discoveries among all discoveries (FDR). We remark that our approach works for any fixed itemset size $k$, unilike traditional frequent itemset mining, where itemsets of all sizes are extracted for a given threshold.

To grasp the intuition behind the above approach, recall that a Poisson distribution models the number of occurrences among a large set of possible events, where the probability of each event is small. In the context of frequent itemset mining, the Poisson approximation holds when the probability that an individual itemset has support at least $s_{\min }$ in $\hat{\mathcal{D}}$ is small, and thus the existence of such event in $\mathcal{D}$ is likely to be statistically significant. We stress that our technique discovers statistically significant itemsets among those of relatively high support. In fact, if the expected supports of individual itemsets vary in a large range, there may exist itemsets with very low expected supports in $\hat{\mathcal{D}}$ which may have statistically significant supports in $\mathcal{D}$. These itemsets would not be discovered by our strategy. However, any mining strategy aiming at discovering significant, low-support itemsets is likely to incur high costs due to the large (possibly exponential) number of candidates to be examined, although only a few of them would turn out to be significant.

We validate our theoretical results by mining significant frequent itemsets from a number of real datasets that are standard benchmarks in this field. Also, we compare the performance of our methodology to a standard multi-hypothesis approach based on [5], and provide evidence that the latter often returns fewer significant itemsets, which indicates that our method has higher power.

\subsection{Related Work}

A number of works have explored various notions of significant itemsets and have proposed methods for their discovery. Below, we review those most relevant to our approach and refer the reader to [15, Section 3] for further references. The paper [1] relates the significance of an itemset $X$ to the quantity $((1-v(X)) /(1-\mathbf{E}[v(X)])) \cdot(\mathbf{E}[v(X)] / v(X))$, where $v(X)$ represents the fraction of transactions containing some but not all of the items of $X$, and $\mathbf{E}[v(X)]$ represents the expectation of $v(X)$ in a random dataset where items occur in transactions independently. This ratio provides an empirical measure of the correlation among the items of $X$ that, according to [1], is more effective than absolute support. In $[9,10,26]$, the significance of an itemset is measured as the ratio $R$ between its actual support and its expected support in a random dataset. In order to make this measure more accurate for small supports, $[9,10]$ proposes smoothing the ratio $R$ using an empirical Bayesian approach. Bayesian analysis is also employed in [24] to derive subjective measures of significance of patterns (e.g., itemsets) based on how strongly they "shake" a system of established beliefs. In [17], the significance of an itemset is defined as the absolute difference between the support of the itemset in the dataset, and the estimate of this support made from a Bayesian network with parameters derived from the dataset.

A statistical approach for identifying significant itemsets is 
presented in [25], where the measure of interest for an itemset is defined as the degree of dependence among its constituent items, which is assessed through a $\chi^{2}$ test. Unfortunately, as reported in $[9,10]$, there are technical flaws in the applications of the statistical test in [25]. Nevertheless, [25] pioneered the quest for a rigorous framework for addressing the discovery of significant itemsets.

A common drawback of the aforementioned works is that they assess the significance of each itemset in isolation, rather than taking into account the global characteristics of the dataset from which they are extracted. As argued before, if the number of itemsets considered by the analysis is large, even in a purely random dataset some of them are likely to be flagged as significant if considered in isolation. A few works attempt at accounting for the global structure of the dataset in the context of frequent itemset mining. The authors of [11] propose an approach based on Markov chains to generate a random dataset that has identical transaction lengths and identical frequencies of the individual items as the given real dataset. The work suggests comparing the outcomes of a number of data mining tasks, frequent itemset mining among the others, in the real and the randomly generated datasets in order to establish whether the real datasets exhibit any significant global structure. However, such an assessment is carried out in a purely qualitative fashion without rigorous statistical grounding.

The problem of spurious discoveries when mining significant patterns is studied in [6]. The paper is concerned with the discovery of significant pairs of items, where significance is measured through the $p$-value, that is, the probability of occurrence of the observed support in a random dataset. Significant pairs are those whose $p$-values are below a certain threshold that can be suitably chosen to bound the FWER, or to bound the FDR. The authors compare the relative power of the two metrics through experimental results, but do not provide methods to set a meaningful support threshold, which is the most prominent feature of our approach.

Beyond frequent itemset mining, the issue of significance has also been addressed in the realm of discovering association rules. In [14], the authors provide a variation of the well-known Apriori strategy for the efficient discovery of a subset $\mathcal{A}$ of association rules with $p$-value below a given cutoff value, while the results in [19] provide the means of evaluating the FDR in $\mathcal{A}$. The FDR metric is also employed in [29] in the context of discovering significant quantitative rules, a variation of association rules. None of these works is able to establish support thresholds such that the returned discoveries feature small FDR.

\subsection{Benchmark datasets}

In order to validate the methodology, a number of experiments, whose results are reported in Section 4, have been performed on datasets which are standard benchmarks in the context of frequent itemsets mining. The main characteristics of the datasets we use are summarized in Table 1. A description of the datasets can be found in the FIMI Repository (http://fimi.cs.helsinki.fi/data/),

\begin{tabular}{|l|c|c|c|c|}
\hline Dataset & $n$ & {$\left[f_{\min } ; f_{\max }\right]$} & $m$ & $t$ \\
\hline \hline Retail & 16470 & {$[1.13 \mathrm{e}-05 ; 0.57]$} & 10.3 & 88162 \\
\hline Kosarac & 41270 & {$[1.01 \mathrm{e}-06 ; 0.61]$} & 8.1 & 990002 \\
\hline Bms1 & 497 & {$[1.68 \mathrm{e}-05 ; 0.06$} & 2.5 & 59602 \\
\hline Bms2 & 3340 & {$[1.29 \mathrm{e}-05 ; 0.05]$} & 5.6 & 77512 \\
\hline Bmspos & 1657 & {$[1.94 \mathrm{e}-06 ; 0.60]$} & 7.5 & 515597 \\
\hline Pumsb* & 2088 & {$[2.04 \mathrm{e}-05 ; 0.79]$} & 50.5 & 49046 \\
\hline
\end{tabular}

Table 1: Parameters of the benchmark datasets: $n$ is the number of items; $\left[f_{\min }, f_{\max }\right]$ is the range of frequencies of the individual items; $m$ is the average transaction length; and $t$ is the number of transactions.

where they are available for download.

\subsection{Organization of the Paper}

The rest of the paper is structured as follows. Section 2 presents the Poisson approximation result for the random variable $\hat{Q}_{k, s}$. The methodology for establishing the support threshold $s^{*}$ is presented in Section 3, and experimental results are reported in Section 4. Section 5 ends the paper with some concluding remarks.

\section{Poisson Approximation for $\hat{Q}_{k, s}$}

The Chen-Stein method [3] is a powerful tool for bounding the error in approximating probabilities associated with a sequence of dependent events by a Poisson distribution. To apply the method to our case, we fix parameters $k$ and $s$, and define a collection of Bernoulli random variables $\left\{Z_{X} \mid X \subset\right.$ $\mathcal{I},|X|=k\}$, such that $Z_{X}=1$ if the itemset $X$ appears in at least $s$ transactions in the random dataset $\hat{\mathcal{D}}$, and $Z_{X}=0$ otherwise. Also, let $p_{X}=\operatorname{Pr}\left(Z_{X}=1\right)$. We are interested in the distribution of $\hat{Q}_{k, s}=\sum_{X:|X|=k} Z_{X}$.

For each set $X$ we define the neighborhood set of $X$,

$$
I(X)=\left\{X^{\prime}\left|X \cap X^{\prime} \neq \emptyset,\right| X^{\prime}|=| X \mid\right\} .
$$

If $Y \notin I(X)$ then $Z_{Y}$ and $Z_{X}$ are independent. Adapting [3, Theorem 1] to our case we have:

Theorem 1. Let $U$ be a Poisson random variable such that $\mathbf{E}[U]=\mathbf{E}\left[\hat{Q}_{k, s}\right]=\lambda<\infty$. The variation distance between the distributions $\mathcal{L}\left(\hat{Q}_{k, s}\right)$ of $\hat{Q}_{k, s}$ and $\mathcal{L}(U)$ of $U$ is such that

$$
\begin{aligned}
\left\|\mathcal{L}\left(\hat{Q}_{k, s}\right)-\mathcal{L}(U)\right\| & =\sup _{A}\left|\operatorname{Pr}\left(\hat{Q}_{k, s} \in A\right)-\operatorname{Pr}(U \in A)\right| \\
& \leq b_{1}+b_{2},
\end{aligned}
$$

where

$$
b_{1}=\sum_{X:|X|=k} \sum_{Y \in I(X)} p_{X} p_{Y}
$$

and

$$
b_{2}=\sum_{X:|X|=k} \sum_{X \neq Y \in I(X)} \mathbf{E}\left[Z_{X} Z_{Y}\right] .
$$


It is easy to see that the quantities $b_{1}$ and $b_{2}$ in the above theorem are both decreasing in $s$. Therefore, if $b_{1}+b_{2}<$ $\epsilon$ for a given $s$, then the same upper bound will hold for every $s^{\prime}>s$. Consequently, a given Poisson approximation for $\hat{Q}_{k, s}$, established through the above theorem, extends to $\hat{Q}_{k, s^{\prime}}$ with $s^{\prime}>s$.

We can derive analytic bounds for $b_{1}$ and $b_{2}$ in many situations. Specifically, suppose that we generate $t$ transactions in the following way. For each item $x$, we sample a random variable $R_{x} \in[0,1]$ independently from some distribution $R$. Conditioned on the $R_{x}$ 's, each item $x$ occurs independently in each transaction with probability $R_{x}$. In what follows, we provide specific bounds for this situation that depend on the moment $\mathbf{E}\left[R^{2 s}\right]$ of the random variable $R$.

Theorem 2. Consider an asymptotic regime where as $n \rightarrow$ $\infty$, we have $k, s=O(1)$ with $s \geq 2, \mathbf{E}\left[R^{2 s}\right]=O\left(n^{-a}\right)$ for some constant $2<a \leq 2 s$, and $t=O\left(n^{c}\right)$ for some positive constant $c$. If $c \leq((k-1)(a-2)+\min (2 a-6,0)) /(2 s)$, then the variation distance between the distributions $\mathcal{L}\left(\hat{Q}_{k, s}\right)$ and $\mathcal{L}(U)$ of $\hat{Q}_{k, s}$ and $U$ satisfies

$$
\begin{aligned}
\left\|\mathcal{L}\left(\hat{Q}_{k, s}\right)-\mathcal{L}(U)\right\| & =\sup _{A}\left|\operatorname{Pr}\left(\hat{Q}_{k, s} \in A\right)-\operatorname{Pr}(U \in A)\right| \\
& =O(1 / n)
\end{aligned}
$$

ProOF. Applying Theorem 1 gives

$$
\left\|\mathcal{L}\left(\hat{Q}_{k, s}\right)-\mathcal{L}(U)\right\| \leq b_{1}+b_{2}
$$

where

$$
b_{1}=\sum_{X:|X|=k} \sum_{Y \in I(X)} p_{X} p_{Y}
$$

and

$$
b_{2}=\sum_{X:|X|=k} \sum_{Y \neq X \in I(X)} \mathbf{E}\left[Z_{X} Z_{Y}\right]
$$

We now evaluate $b_{1}$ and $b_{2}$. Letting $\vec{R}$ denote the vector of the $R_{x}$ 's, we have that for any set $X$ of $k$ items

$$
\operatorname{Pr}\left(Z_{X}=1 \mid \vec{R}\right) \leq\left(\begin{array}{l}
t \\
s
\end{array}\right) \prod_{x \in X} R_{x}^{s}
$$

Since the $R_{x}$ 's are independent with common distribution $R$,

$$
p_{X}=\mathbf{E}\left[\operatorname{Pr}\left(Z_{X}=1 \mid \vec{R}\right)\right] \leq\left(\begin{array}{l}
t \\
s
\end{array}\right) \mathbf{E}\left[R^{s}\right]^{k}
$$

Using Jensen's inequality, we now have

$$
\begin{aligned}
b_{1} & =\sum_{X:|X|=k} \sum_{Y \in I(X)} p_{X} p_{Y} \\
& \leq\left(\left(\begin{array}{l}
n \\
k
\end{array}\right)^{2}-\left(\begin{array}{c}
n \\
k
\end{array}\right)\left(\begin{array}{c}
n-k \\
k
\end{array}\right)\right)\left(\begin{array}{l}
t \\
s
\end{array}\right)^{2} \mathbf{E}\left[R^{s}\right]^{2 k} \\
& \leq\left(\begin{array}{l}
n \\
k
\end{array}\right)^{2}\left(1-\frac{\left(\begin{array}{c}
n-k \\
k
\end{array}\right)}{\left(\begin{array}{c}
n \\
k
\end{array}\right)}\right)\left(\begin{array}{c}
t \\
s
\end{array}\right)^{2} \mathbf{E}\left[R^{2 s}\right]^{k} \\
& =\left(\begin{array}{l}
n \\
k
\end{array}\right)^{2}\left(1-\prod_{i=0}^{k-1} \frac{n-k-i}{n-i}\right)\left(\begin{array}{l}
t \\
s
\end{array}\right)^{2} \mathbf{E}\left[R^{2 s}\right]^{k} \\
& =\Theta\left(n^{k}\right)^{2} \cdot \Theta(1 / n) \cdot O\left(n^{2 c s}\right) \cdot O\left(n^{-k a}\right) \\
& =O\left(n^{k(2-a)+2 c s-1}\right)
\end{aligned}
$$

We now turn our attention to $b_{2}$. Consider sets $X \neq Y$ of $k$ items, and suppose $g=|X \cap Y|>0$. If $Z_{X} Z_{Y}=1$, there exist disjoint subsets $A, B, C \in\{1, \ldots, t\}$ such that $0 \leq|A| \leq s,|B|=|C|=s-|A|$, all of the transactions in $A$ contain both $X$ and $Y$, all of the transactions in $B$ contain $X$, and all of the transactions in $C$ contain $Y$. Therefore,

$$
\begin{aligned}
\mathbf{E}\left[Z_{X} Z_{Y} \mid \vec{R}\right] \leq \sum_{i=0}^{s}\left(\begin{array}{c}
t \\
i ; s-i ; s-i
\end{array}\right)\left(\prod_{x \in X \cup Y} R_{x}^{i}\right) & \times\left(\prod_{x \in X} R_{x}^{s-i}\right)\left(\prod_{y \in Y} R_{y}^{s-i}\right) \\
= & \sum_{i=0}^{s}\left(\begin{array}{c}
t \\
i ; s-i ; s-i
\end{array}\right)\left(\prod_{x \in X \cap Y} R_{x}^{2 s-i}\right) \\
& \times\left(\prod_{x \in X-Y} R_{x}^{s}\right)\left(\prod_{y \in Y-X} R_{y}^{s}\right) .
\end{aligned}
$$

Applying independence of the $R_{x}$ 's and Jensen's inequality gives

$$
\begin{aligned}
\mathbf{E}\left[Z_{X} Z_{Y}\right] & =\mathbf{E}\left[\mathbf{E}\left[Z_{X} Z_{Y} \mid \vec{R}\right]\right] \\
& \leq \sum_{i=0}^{s}\left(\begin{array}{c}
t \\
i ; s-i ; s-i
\end{array}\right) \mathbf{E}\left[R^{2 s-i}\right]^{g} \mathbf{E}\left[R^{s}\right]^{2(k-g)} \\
& \leq \sum_{i=0}^{s} t^{2 s-i} \mathbf{E}\left[R^{2 s}\right]^{\frac{g(2 s-i)}{2 s}} \mathbf{E}\left[R^{2 s}\right]^{k-g} \\
& =\sum_{i=0}^{s} t^{2 s-i} \mathbf{E}\left[R^{2 s}\right]^{k-i g / 2 s} \\
& \leq O(1) \sum_{i=0}^{s} n^{(2 s-i) c-a(k-i g / 2 s)} \\
& =O\left(n^{2 s c-a k}\right) \sum_{i=0}^{s} n^{i\left(\frac{a g}{2 s}-c\right)} \\
& =O\left(n^{2 s c-a k+\max \left\{0, s\left(\frac{a g}{2 s}-c\right)\right\}}\right)
\end{aligned}
$$


It follows that

$$
\begin{aligned}
b_{2} & \leq \sum_{g=1}^{k-1}\left(\begin{array}{c}
n \\
g ; k-g ; k-g
\end{array}\right) O\left(n^{2 s c-a k+\max \left\{0, s\left(\frac{a g}{2 s}-c\right)\right\}}\right) \\
& =O\left(n^{2 k+2 s c-a k}\right) \sum_{g=1}^{k-1} n^{-g} O\left(n^{\max \left\{0, s\left(\frac{a g}{2 s}-c\right)\right\}}\right)
\end{aligned}
$$

Now, for $2 s c / a<g<k$, we have (using the fact that $a \geq 2$ )

$$
n^{-g} n^{\max \left\{0, s\left(\frac{a g}{2 s}-c\right)\right\}}=n^{g\left(\frac{a}{2}-1\right)-s c} \leq n^{(k-1)\left(\frac{a}{2}-1\right)-s c} .
$$

Thus, $b_{2}=O\left(n^{2 k+s c-a k+(k-1)\left(\frac{a}{2}-1\right)}\right)$. (Here we are using the fact that our choice of $c$ satisfies $c \leq(k-1)(a-2) / 2 s$ to ensure that $n^{(k-1)\left(\frac{a}{2}-1\right)-c s}=\Omega(1)$.)

Now, we have $b_{1}=O(1 / n)$ since $c \leq(k-1)(a-2) / 2 s \leq$ $k(a-2) / 2 s$, and $b_{2}=O(1 / n)$ since $c \leq[k(a-2)+(a-$ $4)] / 2 s$. Thus, $b_{1}+b_{2}=O(1 / n)$.

\subsection{A Monte Carlo method for determining $s_{\min }$}

Theorem 2 provides rigorous analytical evidence that there exists a meaningful range for the support $s$ such that the number of itemsets of size $k$ with support $s$ or larger can be approximated by a Poisson variable. Observe that the theorem applies to random datasets satisfying a slightly more stringent condition on the individual item frequencies. In what follows, we give experimental evidence that the result holds for our reference random model by establishing $s_{\text {min }}$ via a simple Monte Carlo simulation which estimates the values $b_{1}$ and $b_{2}$ as defined in Theorem 1. This approach is also convenient in practice since it avoids the inevitable slack due to the use of asymptotics in Theorem 2

For clarity, we use the notation $b_{1}(s)$ and $b_{2}(s)$ to indicate explicitly that both quantities are functions of the support $s$. Suppose that for a chosen $\epsilon, 0<\epsilon<1$, we want to determine $s_{\min }=\min \left\{s \geq 1: b_{1}(s)+b_{2}(s) \leq \epsilon\right\}$. Let $\tilde{s}$ be the maximum expected support of any $k$-itemset (we expect $\tilde{s}<s_{\min }$ ). We generate $\Delta$ random datasets and extract, from each such dataset, all $k$-itemsets of support at least $\tilde{s}$. Let $W$ be the set of itemsets extracted in this fashion from all the generated datasets. It is easy to see that for each $s \geq \tilde{s}$ we can estimate $b_{1}(s)$ and $b_{2}(s)$ by computing for each $X \in W$ the empirical probability $p_{X}$ of the event $Z_{X}=1$, and for each pair $X, Y \in W$, with $X \cap Y \neq \emptyset$, the empirical probability $p_{X, Y}$ of the event $\left(Z_{X}=1\right) \wedge\left(Z_{Y}=1\right)$. Note that for itemsets not in $W$ these probabilities are estimated as 0 . Then, if it turns out that $b_{1}(\tilde{s})+b_{2}(\tilde{s})>\epsilon$, we let $s_{\text {min }}$ be the minimum $s>\tilde{s}$ such that $b_{1}(s)+b_{2}(s) \leq \epsilon$. Otherwise, if $b_{1}(\tilde{s})+b_{2}(\tilde{s}) \leq \epsilon$, we repeat the above procedure starting from $\tilde{s} / 2$. It can be shown (details omitted for lack of space) that for $\Delta=O(\log (1 / \delta) / \epsilon)$, the output $s_{\min }$ of the MonteCarlo process satisfies $\operatorname{Pr}\left(b_{1}\left(s_{\min }\right)+b_{2}\left(s_{\min }\right) \leq \epsilon\right) \geq 1-\delta$. Algorithm 1 implements the above ideas. We remark that the information mined from the random datasets can also be used to obtain an estimate $\hat{\lambda}(s)$ of the expected number $\lambda(s)$ of itemsets with support at least $s$ for every $s \geq s_{\min }$, that

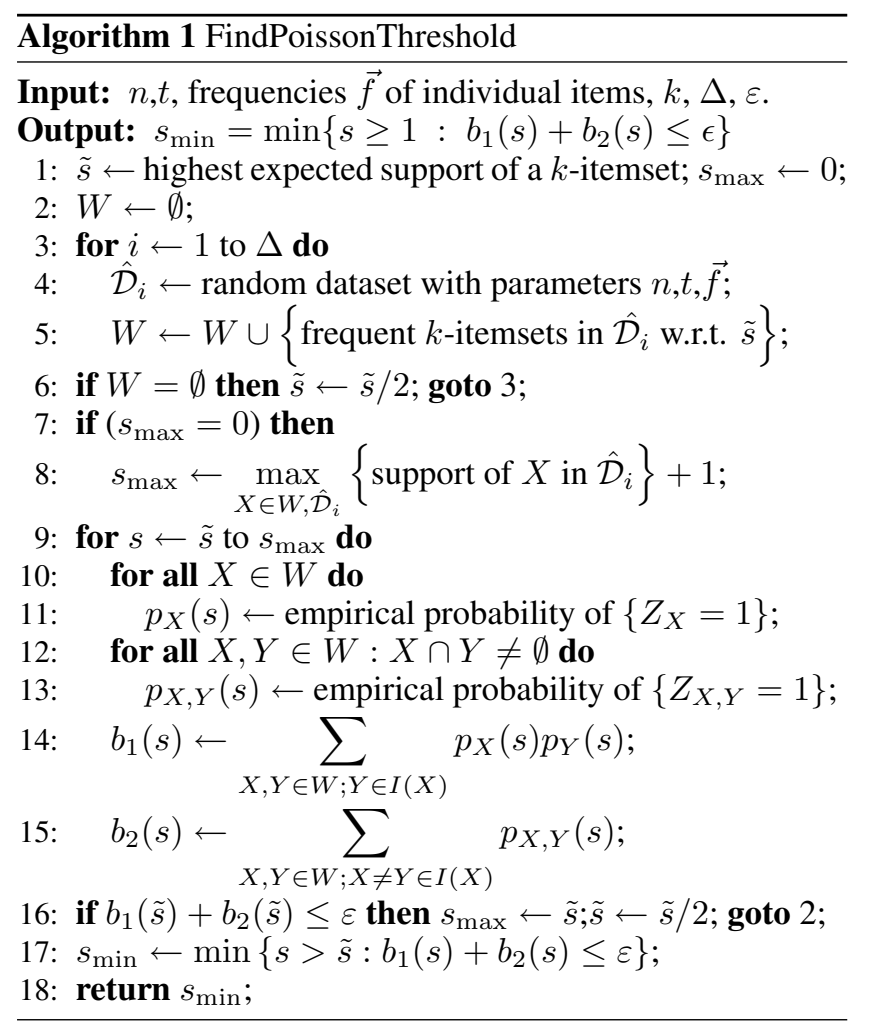

is the expectation of the Poisson distribution of $\hat{Q}_{k, s}$. We denote the set of values $\left\{\lambda(s): s \geq s_{\min }\right\}$ by the vector $\vec{\lambda}$. These values are needed to perform our statistical tests illustrated in the next section.

For each dataset $\mathcal{D}$ of Table 1 and for itemset sizes $k=$ 2, 3, 4, we applied Algorithm 1 setting $\Delta=1000$ and $\epsilon=$ 0.01 . The values of $s_{\min }$ we obtained are reported in Table 2 (we added the prefix "Rand" to each dataset name, to denote the fact that the dataset is random and features the same parameters as the corresponding real one).

\begin{tabular}{|l|c||c||c|}
\hline \multirow{2}{*}{ Dataset } & \multicolumn{3}{|c|}{$s_{\min }$} \\
\cline { 2 - 4 } & $k=2$ & $k=3$ & $k=4$ \\
\hline \hline RandRetail & 9237 & 4366 & 784 \\
\hline RandKosarak & 273266 & 100543 & 20120 \\
\hline RandBms1 & 268 & 23 & 5 \\
\hline RandBms2 & 168 & 13 & 4 \\
\hline RandBmspos & 76672 & 15714 & 2717 \\
\hline RandPumsb* & 29303 & 21893 & 16265 \\
\hline
\end{tabular}

Table 2: Minimum support $s_{\min }$ for which the Poisson approximation for $\hat{Q}_{k, s}$ holds (i.e., $b_{1}+b_{2}<0.01$ ) for $k=2,3,4$ in random datasets with the same values of $n, t$, and with the same frequencies of the items as the corresponding benchmark datasets.

\section{Establishing a support threshold for mining statistically significant frequent itemsets}

In this section we describe our testing methodology for 
determining a support threshold $s^{*}$ such that the family of frequent itemsets with respect to $s^{*}$ are statistically significant with a controlled FDR. At the end of the section (Subsection 3.1), we briefly describe a standard multi-comparison test to identify significant itemsets with small FDR, which will be employed in Section 4 as a comparison point to assess the benefits of our methodology.

Let $\mathcal{D}$ be the input dataset and $k$ a fixed itemset size. As before, we use $Q_{k, s}$ to denote the number of itemsets of size $k$ of support at least $s$ in $\mathcal{D}$, and $\hat{Q}_{k, s}$ to denote the corresponding random variable for $\hat{\mathcal{D}}$. We seek a support threshold $s^{*}$ such that the observed value $Q_{k, s^{*}}$ is significantly large compared to the expected value of $\hat{Q}_{k, s^{*}}$, in the sense that the probability that such a large deviation occurs in a random dataset is bounded by a pre-selected constant $\alpha$.

Let $s_{\min }$ be the minimum support such that the Poisson approximation for the distribution of $\hat{Q}_{k, s}$ holds for $s \geq s_{\min }$, and let $s_{\max }$ be the maximum support of an item in $\mathcal{D}$. Our testing methodology performs $h=\left\lfloor\log _{2}\left(s_{\max }-s_{\text {min }}\right)\right\rfloor+1$ comparisons. The null hypothesis $H_{0}^{i}$ in the $i$-th comparison, for $0 \leq i<h$, is that the observed value $Q_{k, s_{i}}$, with $s_{0}=$ $s_{\text {min }}$ and $s_{i}=s_{\text {min }}+2^{i}$ for $1 \leq i<h$, is drawn from the same Poisson distribution as $\hat{Q}_{k, s_{i}}$. For every $0 \leq i<h$ we fix a confidence level $\alpha_{i}$ and reject the null hypothesis $H_{0}^{i}$ if the $p$-value of $Q_{k, s_{i}}$ is smaller than $\alpha_{i}$. If the $\alpha_{i}$ 's are chosen so that $\sum_{i=0}^{h-1} \alpha_{i}=\alpha$, the union bound shows that the probability of rejecting any true null hypothesis is less than $\alpha$.

Suppose we set $s^{*}$ as the minimum of the $s_{i}$ 's for which the null hypothesis $H_{0}^{i}$ was rejected. The probability that this number of itemsets of size $k$ with support at least $s_{i}$ were observed in a random dataset is bounded by $\alpha$, thus the size of this set is statistically significant. While this approach is a useful starting point in itself, it does not imply necessarily that all of these frequent itemsets are statistically significant. In fact, some of them are likely to occur with high support even under $H_{0}^{i}$, and hence they would represent false discoveries. In order to ensure that the FDR is below a specified level $\beta$, we can further amend the above procedure as follows.

Fix suitable values $\beta_{0}, \beta_{1}, \ldots, \beta_{h-1}$ such that $\sum_{i=0}^{h-1} \beta_{i}^{-1} \leq \beta$. For $0 \leq i<h$, let $\lambda_{i}=E\left[\hat{Q}_{k, s_{i}}\right]$. We modify the test described above by rejecting the $i$-th null hypothesis $H_{0}^{i}$ if the probability that a Poisson random variable with expectation $\lambda_{i}$ takes a value as large as the observed $Q_{k, s_{i}}$ is at most $\alpha_{i}$, and $Q_{k, s_{i}} \geq \beta_{i} \lambda_{i}$. Again, we set $s^{*}$ as the minimum of the $s_{i}$ 's for which the null hypothesis $H_{0}^{i}$ was rejected. We now prove that with this variation the FDR of the family of frequent itemsets of size $k$ mined with threshold $s^{*}$ is upper bounded by $\beta$.

We denote by $\mathcal{F}_{(k)}\left(s_{i}\right)$ the family of itemsets of size $k$ with support at least $s_{i}$ in $\mathcal{D}$, and note that $\left|\mathcal{F}_{(k)}\left(s_{i}\right)\right|=Q_{k, s_{i}}$. Let $V_{i}$ be the number of false discoveries if $H_{0}^{i}$ were the first null hypothesis rejected, in which case $\mathcal{F}_{(k)}\left(s_{i}\right)$ would be returned as the family of significant itemsets. Let $E_{i}$ be the condition " $H_{0}^{i}$ is rejected" or equivalently, "the $p$-value of $Q_{k, s_{i}}$ is smaller than $\alpha_{i}$ and $Q_{k, s_{i}} \geq \beta_{i} \lambda_{i}$ ". If a discovery is false positive then its distribution is as in the null hypotheses $H_{0}^{i}$. Thus, the distribution of $V_{i}$ is stochastically bounded by that of a Poisson variable $X_{i}$ with expectation $\lambda_{i}$, conditioned on the events $E_{i}, \bar{E}_{i-1}, \ldots, \bar{E}_{0}$. Therefore,

$$
\begin{aligned}
F D R & =\sum_{i=0}^{h-1} E\left[\frac{V_{i}}{Q_{k, s_{i}}}\right] \operatorname{Pr}\left(E_{i}, \bar{E}_{i-1}, \ldots, \bar{E}_{0}\right) \\
& \leq \sum_{i=0}^{h-1} \frac{E\left[X_{i} \mid E_{i} \bar{E}_{i-1}, \ldots, \bar{E}_{0}\right]}{\beta_{i} \lambda_{i}} \operatorname{Pr}\left(E_{i}, \bar{E}_{i-1}, \ldots, \bar{E}_{0}\right) \\
& =\sum_{i=0}^{h-1} \frac{\sum_{j} j \operatorname{Pr}\left(X_{i}=j, E_{i}, \bar{E}_{i-1}, \ldots, \bar{E}_{0}\right)}{\beta_{i} \lambda_{i}} \\
& \leq \sum_{i=0}^{h-1} \frac{\lambda_{i}}{\beta_{i} \lambda_{i}} \leq \sum_{i=0}^{h-1} \frac{1}{\beta_{i}}
\end{aligned}
$$

Since $\sum_{i=0}^{h-1} \alpha_{i} \leq \alpha$, and $\sum_{i=0}^{h-1} \beta_{i}^{-1} \leq \beta$ we obtain a test that identifies with confidence $1-\alpha$ a minimum support $s^{*}$ such that $\left|\mathcal{F}_{(k)}\left(s^{*}\right)\right|$ is significant. Moreover, the FDR among the individual itemsets of $\mathcal{F}_{(k)}\left(s^{*}\right)$ is bounded by $\beta$. The pseudocode Test 1 specifies more formally our test to determine the support threshold $s^{*}$.

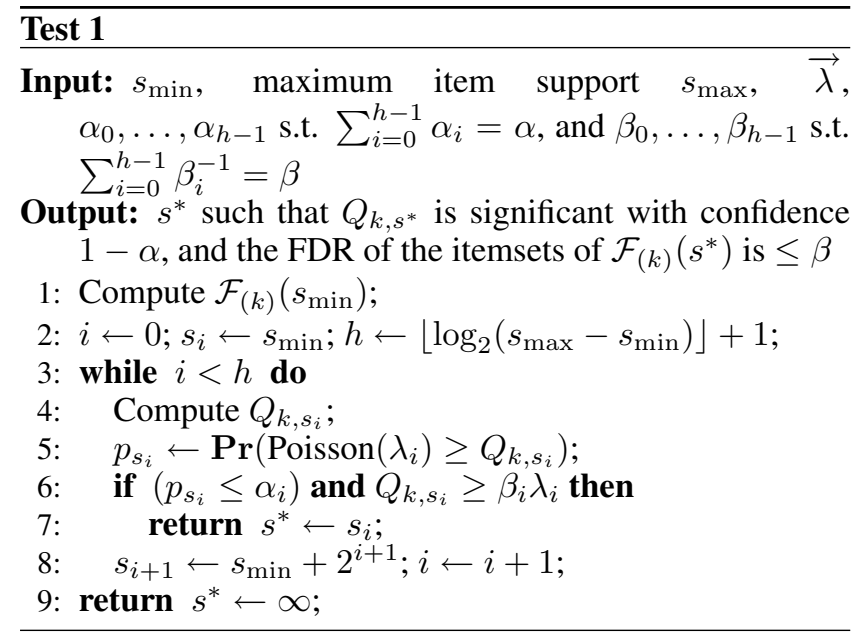

\subsection{A standard multi-comparison test}

To assess the effectiveness of our new approach we will compare it against the following standard multi-comparison test based on the state-of-the-art technique of [5].

Theorem 3 (Benjamini AND YeKuTIELI [5]). Assume that we are testing for $m$ null hypotheses. Let $p_{(1)} \leq p_{(2)} \leq \cdots \leq p_{(m)}$ be the ordered observed $p$-values of the $m$ tests. For control of FDR at level $\beta$, define

$$
\ell=\max \left\{i \geq 0: p_{(i)} \leq \frac{i}{m \sum_{j=1}^{m} \frac{1}{j}} \beta\right\},
$$


and reject the null hypotheses of tests $(1), \ldots,(\ell)$.

As before, let $\mathcal{D}$ denote the input dataset consisting of $t$ transactions over $n$ items. Let $s$ be a given support threshold and $k$ a fixed itemset size. After mining the frequent $k$ itemsets $\mathcal{F}_{(k)}(s)$ we test, for each $X \in \mathcal{F}_{(k)}(s)$, the null hypothesis $H_{0}^{X}$ that the observed support of $X$ in $\mathcal{D}$ is drawn from a Binomial distribution with parameters $t$ and $f_{X}$ (the product of the individual frequencies of the items of $X$ ), setting the rejection threshold as specified by condition (1), with parameters $\beta$ and $m=\left(\begin{array}{l}n \\ k\end{array}\right)$. The itemsets of $\mathcal{F}_{(k)}(s)$ whose associated null hypothesis is rejected can be returned as significant with FDR upper bounded by $\beta$.

The pseudocode Test 2 summarizes the test described above.

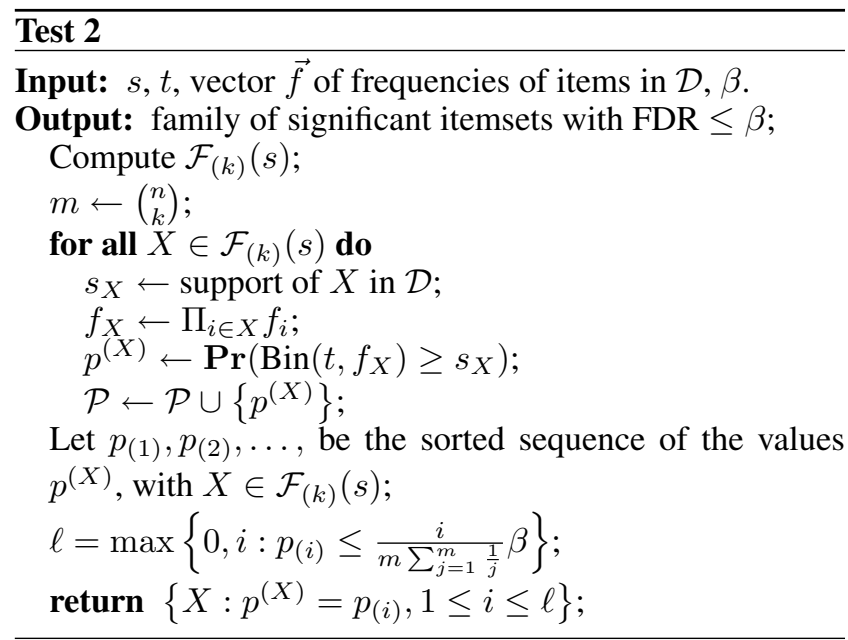

\section{Experimental Results}

In this section, we report on a number of experiments devised to validate and show the potential of our approach. In Subsection 4.1, we apply our methodology to the benchmark datasets of Table 1. In Subsection 4.2, we compare the obtained results against those returned by the standard procedure to bound the FDR described in Subsection 3.1.

\subsection{Experiments on benchmark datasets}

We first apply our methodology to the benchmark datasets of Table 1. Specifically, for each dataset and for $k=2,3,4$, we apply Test 1 to identify a support threshold $s^{*}$ such that the number of $k$-itemsets that appear in at least $s^{*}$ transactions represent a significant deviation from what would be expected in a random dataset, with significance level $\alpha=0.05\left(\alpha_{i}=0.05 / h\right)$, and with FDR of the returned family of itemsets at most $\beta=0.05\left(\beta_{i}^{-1}=0.05 / h\right)$. The results are displayed in Table 3 , where, for each benchmark dataset and for $k=2,3,4$, we show: the minimum support $s^{*}$, if any, for which the corresponding null hypothesis was rejected; the number of itemsets $Q_{k, s^{*}}$ with support at least $s^{*}$; the expected number $\lambda\left(s^{*}\right)$ of itemsets with support at least $s^{*}$ in a corresponding random dataset; and the ratio $r=\lambda\left(s^{*}\right) / Q_{k, s^{*}}$.

We also conducted an almost identical set of experiments, which are not reported in tabular form here for the sake of brevity, where we maintained the confidence level $\alpha=0.05$ for the selection of $s^{*}$, but did not control the FDR (setting all $\beta_{i}$ 's to 0 in Test 1 ). These experiments behaved as one would expect; we observed some decrease in the support threshold required for rejection of the null hypothesis, and a consequent increase in the number of flagged itemsets, at the expense of some increase in the rate of false positive discoveries.

We observe that for most pairs (dataset, $k$ ) the number of significant frequent $k$-itemsets obtained is small, focusing on the most frequent significant itemsets. The results provide evidence that our methodology not only defines significance on statistically rigorous grounds, but also provides the mining task with suitable support thresholds that avoid explosion of the output size (the widely recognized "Achilles' heel" of traditional frequent itemset mining). This crucially relies on the identification of a region of "rare events" provided by the Poisson approximation. As discussed in Section 1.3, the discovery of significant itemsets with low support (not returned by our method) would require the extraction of a large (possibly exponential) number of itemsets, that would make any strategy aiming to discover these itemsets unfeasible. Instead we provide an efficient method to identify with high confidence level the family of most frequent itemsets that are statistically significant without overwhelming the user with a huge number of discoveries.

There are, however, a few cases where the number of itemsets returned is still considerably high. Their large number often serves as a sign that the results call for further analysis, possibly using clustering techniques [28] or searching for closed representatives for these itemsets [21]. For example, considering dataset Bms1 with $k=4$ and the corresponding value $s^{*}=5$ from Table 3 . Extracting the closed itemsets of support greater or equal to $s^{*}$ in that dataset revealed the presence of a closed itemset of cardinality 154 that appears more than 7 times in the dataset. This itemset, whose occurrence by itself represents an extremely unlikely event in a random dataset, accounts for more than $22 \mathrm{M}$ itemsets among the $27 \mathrm{M}$ reported as significant.

It is interesting to observe that the results obtained for dataset Retail provide further evidence for the conclusions drawn in [11], which suggests random behavior for this dataset (although the random model in that work is slightly different from ours, in that the family of random datasets also maintains the same transaction lengths as the real one). Indeed, no support threshold $s^{*}$ could be established for mining significant $k$-itemsets with $k=2,3$, while the support threshold $s^{*}$ identified for $k=4$ yielded only 6 itemsets. However, the conclusion drawn in [11] was based on a qualitative assessment of the discrepancy between the numbers of frequent itemsets in the random and real datasets, while our methodology confirms the findings on a statistically sound and rigorous basis. 


\begin{tabular}{|l|c|c|c|c||c|c|c|c||c|c|c|c|}
\hline \multirow{2}{*}{ Dataset } & \multicolumn{9}{|c||}{$k=2$} & \multicolumn{4}{c||}{$k=3$} & \multicolumn{4}{c|}{$k=4$} \\
\cline { 2 - 14 } & $s^{*}$ & $Q_{k, s^{*}}$ & $\lambda\left(s^{*}\right)$ & $r$ & $s^{*}$ & $Q_{k, s^{*}}$ & $\lambda\left(s^{*}\right)$ & $r$ & $s^{*}$ & $Q_{k, s^{*}}$ & $\lambda\left(s^{*}\right)$ & $r$ \\
\hline \hline Retail & - & - & - & - & - & - & - & - & 848 & 6 & 0.01 & 0.0017 \\
\hline Kosarak & - & - & - & - & - & - & - & - & 21144 & 12 & 0.01 & 0.0008 \\
\hline Bms1 & 276 & 56 & 0.19 & 0.0034 & 23 & 258859 & 0.06 & $2 \times 10^{-7}$ & 5 & $27 \mathrm{M}$ & 0.05 & $2 \times 10^{-9}$ \\
\hline Bms2 & 168 & 429 & 0.73 & 0.0017 & 13 & 36112 & 0.25 & $7 \times 10^{-6}$ & 4 & 714045 & 0.01 & $1 \times 10^{-8}$ \\
\hline Bmspos & - & - & - & - & 16226 & 24 & 0.01 & 0.0005 & 2717 & 891 & 0.38 & 0.0004 \\
\hline Pumsb & 29303 & 29 & 0.05 & 0.0017 & 21893 & 406 & 0.35 & 0.0009 & 16265 & 6293 & 1.37 & 0.0002 \\
\hline
\end{tabular}

Table 3: Results for Test 1 with $\alpha=0.05, \beta=0.05$.

Observe also that for some other pairs (dataset, $k$ ) our tests do not find any support threshold useful to identify statistically significant itemsets. This is an evidence that, for the specific $k$ and for the supports considered by our tests, these datasets do not present a significant deviation from a corresponding random dataset.

In order to assess its robustness, we applied our methodology to random datasets. Specifically, for each benchmark dataset of Table 1 and for $k=2,3,4$, we generated 100 random instances with the same parameters as those of the benchmark, and applied Test 1 to each instance searching for a support threshold $s^{*}$ for mining significant itemsets. As expected, the test was not able to reject any null hypothesis, hence it did not determine $s^{*}$, in all cases but for 2 of the 100 instances of the random dataset with the same parameters as dataset Pumsb* with $k=2$. However, in these two cases, mining at the identified support threshold only yielded as few as 1 and 2 itemsets, respectively.

\subsection{Comparison with the Standard FDR Test (Test 2)}

We compare the number of itemsets extracted using the threshold $s^{*}$ provided by Test 1 , with the number of itemsets flagged as significant using the standard method described in Section 3.1 using the same threshold $s^{*}$. In both cases we imposed a bound $\beta=0.05$ on the FDR.

The results are displayed in Table 4, where for each pair (dataset, $k$ ), we report the cardinality of the family $\mathcal{R}$ of significant itemsets returned by the method of Section 3.1, and the ratio $r=|\mathcal{R}| / Q_{k, s^{*}}$, where $Q_{k, s^{*}}$ is the number of significant itemsets returned by our methodology. We observe that in some cases the method of Section 3.1 identifies a small fraction of the itemsets flagged as significant by Test 1 . Indeed, in two cases more than $90 \%$ of the significant itemsets returned by our methodology are not flagged as significant by the standard method. Since we imposed a bound $\beta=0.05$ on the FDR for both tests, the itemsets not identified by the method of Section 3.1 correspond in large part to significant itemsets. Test 1 succeeds in identifying these itemsets, since it evaluates the significance of the entire set of itemsets of support $s^{*}$ comparing $Q_{k, s^{*}}$ to $\hat{Q}_{k, s^{*}}$. In contrast, Test 2 has to control for testing of significantly more hypothesis (corresponding to the significance all possible $k$-itemsets), thus the power of the test $(1-\operatorname{Pr}$ (Type-II error)) is significantly smaller. These results demonstrate the advantage of our technique compared to the standard approach for multihypothesis tests that control FDR.

\begin{tabular}{|l|c|c||c|c||c|c|}
\hline \multirow{2}{*}{ Dataset } & \multicolumn{2}{|c||}{$k=2$} & \multicolumn{2}{c||}{$k=3$} & \multicolumn{2}{c|}{$k=4$} \\
\cline { 2 - 7 } & $|\mathcal{R}|$ & $r$ & $|\mathcal{R}|$ & $r$ & $|\mathcal{R}|$ & $r$ \\
\hline \hline Retail & - & - & - & - & 6 & 1.0 \\
\hline Kosarak & - & - & - & - & 12 & 1.0 \\
\hline Bms1 & 55 & 0.982 & 64367 & 0.249 & 219706 & 0.008 \\
\hline Bms2 & 429 & 1.0 & 25906 & 0.717 & 60927 & 0.085 \\
\hline Bmspos & - & - & 24 & 1.0 & 891 & 1.0 \\
\hline Pumsb & 29 & 1.0 & 406 & 1.0 & 6288 & 0.999 \\
\hline
\end{tabular}

Table 4: Results using Test 2 to bound the FDR with $\beta=$ 0.05 for itemsets of support $\geq s^{*}$.

\section{Conclusions}

The main technical contribution of the paper is the proof that in a random dataset where items are placed independently in transactions, there is a minimum support $s_{\min }$ such that the number of $k$-itemsets with support at least $s_{\min }$ is well approximated by a Poisson distribution. The expectation of the Poisson distribution and the threshold $s_{\min }$ are functions of the number of transactions, number of items, and frequencies of individual items.

This result is at the base of a novel methodology for mining frequent itemsets which can be flagged as statistically significant incurring a small FDR. In particular, we use the Poisson distribution as the distribution of the null hypothesis in a novel multi-hypothesis statistical approach for identifying a suitable support threshold $s^{*} \geq s_{\min }$ for the mining task. We control the FDR of the output in a way which takes into account global characteristics of the dataset, hence it turns out to be more powerful than other standard statistical tools (e.g., [5]). The results of a number of experiments, reported in the paper, provide evidence of the effectiveness of our approach.

To the best of our knowledge, our methodology represents the first attempt at establishing a support threshold for the classical frequent itemset mining problem with a quantitative guarantee on the significance of the output.

\section{References}

[1] C.C. Aggarwal and P.S. Yu. A new framework for itemset generation. In Proc. of the 17th ACM Symp. on Principles of Database Systems, pages 18-24, 1998. 
[2] R. Agrawal, T. Imielinski, and A.N. Swami. Mining association rules between sets of items in large databases. In Proc. of the ACM SIGMOD Intl. Conference on Management of Data, pages 207-216, 1993.

[3] R. Arratia, L. Goldstein, and L. Gordon. Poisson approximation and the Chen-Stein method. Statistical Science, 5(4):403-434, 1990.

[4] Y. Benjamini, and Y. Hochberg. Controlling the false discovery rate. J. Royal Statistical Society, Series B, 57:289-300, 1995.

[5] Y. Benjamini, and D. Yekutieli The control of the false discovery rate in multiple testing under dependency Annals of Statistics, 29 (4): 1165-1188, 2001.

[6] R.J. Bolton, D.J. Hand, and N.M. Adams. Determining Hit Rate in Pattern Search In Proc. of Pattern Detection and Discovery, LNAI 2447, pages 36-48, 2002.

[7] W. J. Conover. Practical Nonparametric Statistics. Wiley Series in Probability, 3rd Ed., 1999.

[8] S. Dudoit, J. P. Shaffer, and J. C. Boldrick. Multiple hypothesis testing in microarray experiments. Statistical Science, Vol. 18, No. 1, 2003, p. 71-103.

[9] W. DuMouchel. Bayesian data mining in large frequency tables, with an application to the FDA spontaneous reporting system. The American Statistician, 53:177-202, 1999.

[10] W. DuMouchel and D. Pregibon. Empirical Bayes screening for multi-item associations. In Proc. of the 7th ACM SIGKDD Intl. Conference on Knowledge Discovery and Data Mining, pages 67-76, 2001.

[11] A. Gionis, H. Mannila, T. Mielikäinen, and P. Tsaparas. Assessing data mining results via swap randomization. In Proc. of the 12th ACM SIGKDD Intl. Conference on Knowledge Discovery and Data Mining, pages 167-176, 2006.

[12] B. Goethals, R. Bayardo, and M. J. Zaki, editors. Proc. of the 2nd Workshop on Frequent Itemset Mining Implementations (FIMI04), volume 126. CEUR-WS Workshop On-line Proceedings, November 2004.

[13] B. Goethals and M. J. Zaki, editors. Proc. of the 1st Workshop on Frequent Itemset Mining Implementations (FIMI03), volume 90. CEUR-WS Workshop On-line Proceedings, November 2003.

[14] W. Hämäläinen, and M. Nykänen Efficient discovery of statistically significant association rules In Proc. of the 8th IEEE Intl. Conference on Data Mining, pages 203-212, 2008.

[15] J. Han, H. Cheng, D. Xin, and X. Yan. Frequent pattern mining: Current status and future directions. Data Mining and Knowledge Discovery, 14(1), 2007.
[16] J. Han, and M. Kamber. Data Mining: Concepts and Techniques. Morgan Kaufmann, San Mateo, CA, 2001.

[17] S. Jaroszewicz, and T. Scheffer. Fast discovery of unexpected patterns in data, relative to a Bayesian network. In Proc. of the 11th ACM SIGKDD Intl Conference on Knowledge Discovery in Data Mining, pages 118-127, 2005.

[18] H.O. Lancaster. The Chi-squared Distribution. John Wiley \& Sons, New York NY, 1969.

[19] N. Megiddo, and R. Srikant Discovering predictive association rules. In Proc. of the 4th Intl. Conference on Knowledge Discovery and Data Mining, pages 274-278, 1998.

[20] M. Mitzenmacher and E. Upfal. Probability and Computing: Randomized Algorithms and Probabilistic Analysis. Cambridge University Press, 2005.

[21] N. Pasquier, Y. Bastide, R. Taouil, and L. Lakhal. Discovering frequent closed itemsets for association rules. In Proc. of the 7th Int. Conference on Database Theory, pages 398-416, January 1999.

[22] P. W. Purdom, D. Van Gucht, and D. P. Groth. Average case performance of the apriori algorithm. SIAM J. Computing, 33 (5): 1223-1260, 2004.

[23] B. Sayrafi, D. Van Gucht, and P. W. Purdom. On the effectiveness and efficiency of computing bounds on the support of item-sets in the frequent item-sets mining problem. In Proc. of the 1st Intl. Workshop on Open Source Data Mining, OSDM '05, pages: 46-55, 2005.

[24] A. Silberschatz and A. Tuzhilin. What makes patterns interesting in knowledge discovery systems. IEEE Trans. on Knowledge and Data Engineering, 8(6):970-974, 1996.

[25] C. Silverstein, S. Brin, and R. Motwani. Beyond market baskets: Generalizing association rules to dependence rules. Data Mining and Knowledge Discovery, 2(1):39-68, 1998.

[26] R. Srikant and R. Agrawal. Mining quantitative association rules in large relational tables. In Proc. of the ACM SIGMOD Intl. Conference on Management of Data, pages 1-12, 1996.

[27] P. Tan, M. Steinbach, and V. Kumar. Introduction to Data Mining. Addison Wesley, 2006.

[28] D. Xin, J. Han, X. Yan, and H. Cheng. Mining compressed frequent-pattern sets. In Proc. of the 31st Very Large Data Base Conference, pages 709-720, 2005.

[29] H. Zhang, B. Padmanabhan, and A. Tuzhilin. On the discovery of significant statistical quantitative rules. In Proc. of the 10th ACM SIGKDD Intl. Conference on Knowledge Discovery and Data Mining, pages 374-383, 2004. 